\title{
Preparation methods
}

Anatomy, age and growth was measured on more than three individual specimens collected in 2013-2015. The material collected in the field has been stored in $40 \%$ ethanol to avoid drying artefacts. Each species was carefully labeled. The material has been sectioned without embedding and double stained with Safranin/Astrablue with the principal goal to determine the maximum plant tissue age and the stem anatomy just above ground. Therefore, all the sections are anatomically, taxonomically and ecologically comparable. Information on each species is given.

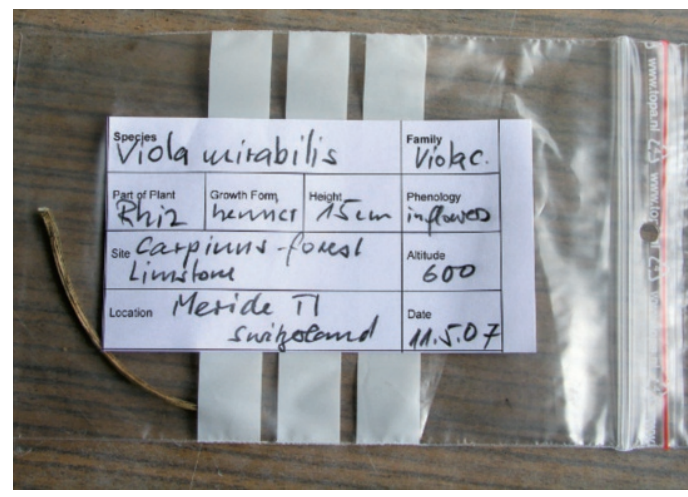

Plastic bag containing $40 \%$ ethanol with a stem of a herb sample. The label contains information about the taxon and its morphology, ecology and the sampling date.

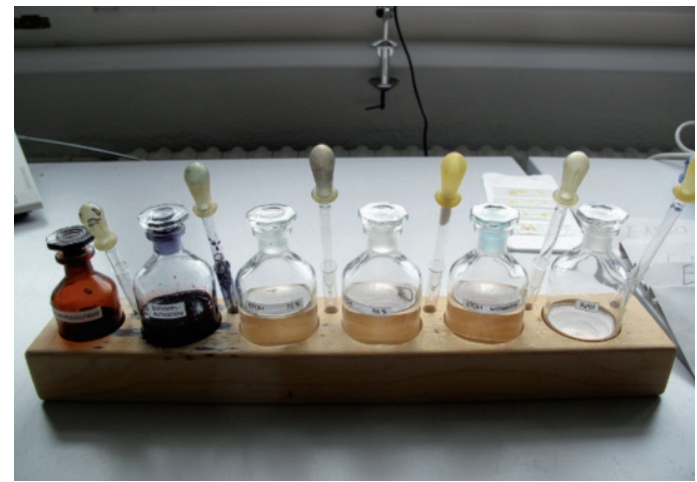

Chemical equipment for staining and dehydration of microsections.

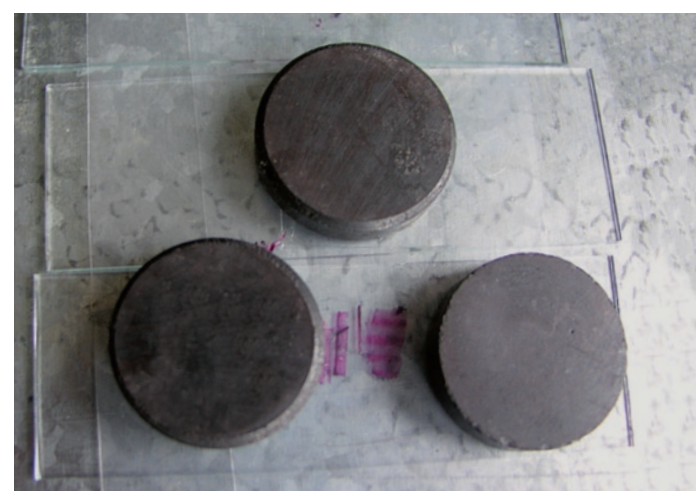

Flattening microscopic sections with magnets on an iron plate.

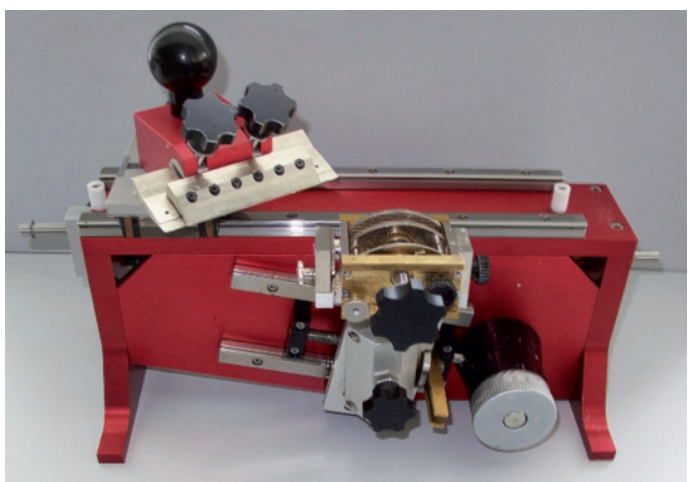

A new laboratory microtome designed by Gaertner et al. 2015.

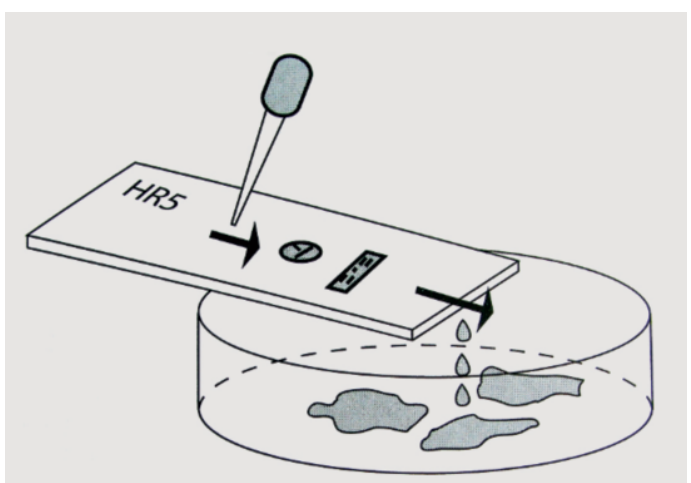

Principle of staining and dehydration on the glass.

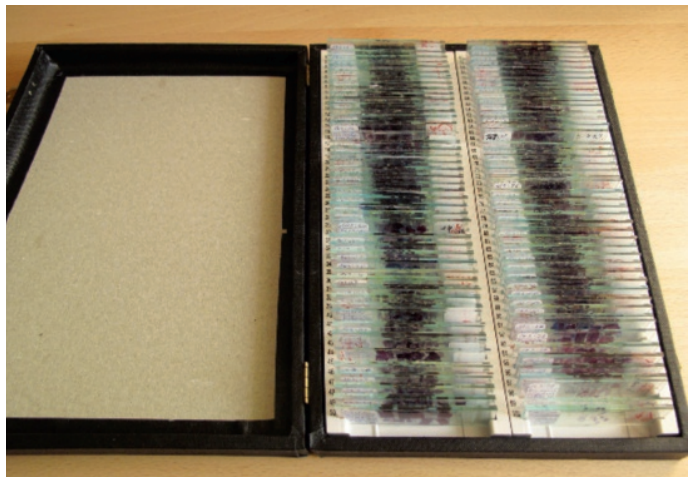

Storing of labeled microscopic slides in boxes. 